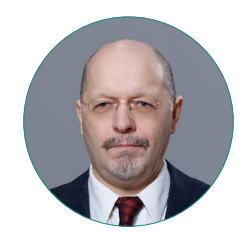

О. Л. Чернозуб

\title{
ДВУХКОМПОНЕНТНАЯ МОДЕЛЬ ПОВЕДЕНИЯ ЧЕЛОВЕКА: МОЖЕТ ЛИ СОЦИОЛОГИЯ ЧЕМУ-ТО НАУЧИТЬ МАРКЕТИНГ?
}

\section{Правильная ссылка на статью:}

Чернозуб О. Л. Двухкомпонентная модель поведения человека: может ли социология чему-то научить маркетинг? // Мониторинг общественного мнения: экономические и социальные перемены. 2021. № 4. С. 77-98. https://doi.org/10.14515/monitoring.2021.4.1946. For citation:

Chernozub O. L. (2021) A Two-Component Model of Behavior Factors: Does Sociology Have Something to Teach Marketing?. Monitoring of Public Opinion: Economic and Social Changes. No. 4. P. 77-98. https://doi.org/10.14515/monitoring.2021.4.1946. (In Russ.) 
ДВУХКОМПОНЕНТНАЯ МОДЕЛЬ ПОВЕДЕНИЯ ЧЕЛОВЕКА: МОЖЕТ ЛИ СОЦИОЛОГИЯ ЧЕМУ-ТО НАУЧИТЬ МАРКЕТИНГ?

ЧЕРНОЗУБ Олег Леонидович - руководитель, Центр социально-экономических исследований, Институт социологии управления, Российская академия народного хозяйства и государственной службы при Президенте РФ, Москва, Россия

E-MAIL: 9166908616@mail.ru

https://orcid.org/0000-0001-5689-8719

Аннотация. В последние годы нарастает волна критики в адрес авторов как политических, так и рыночных прогнозов. Это явление нельзя оценивать иначе как свидетельство несовершенства используемых ими моделей поведения человека. В электоральной социологии, где "цена" ошибок несоизмеримо более высока, стремление избежать их привело к некоторому прогрессу в этой области. Могут ли его результаты быть использованы в маркетинге?

В настоящей статье представлены результаты анализа теоретических предпосылок использования методологических находок электоральной социологии в маркетинге. Также дается метаанализ применения одной из этих находок - двухкомпонентной модели факторов поведения - для прогнозирования результатов голосования. Указанная модель основывается на комбинировании открытых (эксплицитных) и скрытых, или скрываемых (имплицитных), факторов поведения, рассматривая акт поведения как результат разрешения имеющегося между ними конфликта. Отмечая опыт
A TWO-COMPONENT MODEL OF BEHAVIOR FACTORS: DOES SOCIOLOGY HAVE SOMETHING TO TEACH MARKETING?

Oleg L. CHERNOZUB ${ }^{1}$ - Head of Center for Socio-Economic Research, Institute for Sociology of Government E-MAIL:9166908616@mail.ru https://orcid.org/0000-0001-5689-8719

\footnotetext{
${ }^{1}$ Russian Presidential Academy of National Economy and Public Administration, Moscow, Russia
}

Abstract. In recent years, there has been a growing wave of criticism against the authors of both political and market forecasts. This phenomenon testifies to the imperfection of the human behavior models they use. In electoral sociology, where the cost of mistakes is considerably higher, the desire to avoid them has led to some progress. Can its results be used in marketing?

This article presents the analysis of the theoretical prerequisites for using the methodological findings of electoral sociology in marketing. It also provides a meta-analysis of the application of one of these findings, a two-component behavioral model, to predict voting results. This model is based on a combination of open (explicit) and hidden (implicit) factors of behavior, considering the behavioral act as a result of resolving the conflict between them. Noting the experience of applying this model in the political sphere, the author formulates the question: can it be transferred to the field of market research?

The review of the theoretical premises of the observed difficulties shows, that 
применения этой модели в политической сфере, автор задается вопросом: может ли он быть перенесен в область исследований рынка?

Как показывает рассмотрение теоретических предпосылок наблюдаемых затруднений, и в электоральном, и в маркетинговом прогнозировании проблемы имеют схожую природу. Это и открывает возможности для встречного заимствования ими методов преодоления данных проблем.

Ключевые слова: маркетинговый прогноз, прогноз спроса, микросоциология, социальное действие, теория поведения, факторы поведения, двухкомпонентная модель факторов поведения, эксплицитные факторы, имплицитные факторы, социальная установка, ГАТО, ТОД/ТБП, IAT, MODE, RIM electoral and marketing forecasting faces problems of similar nature. This opens up opportunities for the reciprocal borrowing of methods to overcome these problems.

Keywords: market forecast, demand forecast, microsociology, social action, behavior theory, behavior factors, dual process model of cognition, two-component model of behavior factors, precursors of behavior, explicit factors implicit factorsattitudestructural theory of attitude, GATO, TOD/TBP, IAT, MODE, RIM

\section{1. Введение}

Мультидисциплинарный подход, используемый в настоящей статье, неизбежно порождает трудности структурирования предлагаемой информации. Рассматривая сходные проблемы экономической науки и социологии, мы поневоле вынуждены переключать внимание с одного предмета на другой, что, конечно, затрудняет целостное восприятие предлагаемого материала. Мы попытаемся хотя бы отчасти преодолеть это затруднение, дав краткий обзор содержания статьи и в явном виде обозначив логику изложения и взаимосвязь рассматриваемых вопросов.

Первый содержательный раздел «Электоральные и маркетинговые исследования: схожие проблемы, единый источник" открывается обзором внешних признаков неблагополучия, характерных для обеих научных дисциплин. Этот обзор ни в коей мере не претендует на полноту рассмотрения проблемы и ограничивается задачей показать, что, во-первых, существуют определенные проблемы, во-вторых, что они замечены не только профессиональным сообществом, но и потребителями соответствующих исследований. Понимаемая таким образом задача позволяет ограничиться рассмотрением только сферы прогнозов социального поведения (электорального и потребительского) как области, где признаки неблагополучия обнаруживаются в наиболее очевидном виде.

Вторая часть этого раздела представляет собой анализ теоретической основы как социологических, так и экономических моделей, лежащих в основе прогнозирования поведения обоих видов. По мнению автора, такой основой является 
умозрительная конструкция "экономического человека", в своем поведении рационально преследующего собственную выгоду и максимизирующего полезность имеющихся в его распоряжении ресурсов. С одной стороны эта единая основа открывает принципиальную возможность продуктивных междисциплинарных заимствований, а с другой - сама по себе содержит ограничения, являющиеся источником по крайней мере какой-то части проблем, сходство которых для обеих наук вполне очевидно.

Завершается первый содержательный раздел обзором теоретических проблем и фундаментальных ограничений моделей поведения "максимизации полезности" с позиции экономической науки, где данные вопросы проработаны не в пример более глубоко ${ }^{1}$.

Следующий раздел “От „экономического поведения“ к „двухкомпонентной модели“" посвящен изложению позитивной теории, претендующей заместить собой модель “экономического поведения",- - двухкомпонентной модели факторов поведения. Ее базовое допущение заключается в том, что в реальности поведение человека определяется не только осознанными мотивами, но и мотивами, находящимися вне пределов контроля сознания субъекта действия. В этих условиях возникает самостоятельная проблема взаимодействия и даже конфликта этих мотивов. Структурная теория установки оказывается удачным инструментом, эффективно описывающим этот конфликт и позволяющим рассматривать совершенное действие как итог разрешения этого конфликта.

Наконец, итоговый раздел “Двухкомпонентная модель: первые успехи в электоральном прогнозировании и некоторые уроки для маркетинга" представляет некоторые первые результаты применения двухкомпонентной модели факторов к прогнозированию электорального поведения в рамках реальных эмпирических исследований 2016-2018 гг. Их результаты свидетельствуют о возникновении устойчивого синергетического эффекта, когда прогноз, учитывающий оба фактора, оказывается более точным, чем прогноз на основе каждого из факторов, используемых изолированно. Раздел завершается обзором предполагаемых сфер применения двухкомпонентной модели факторов поведения в маркетинговом планировании и маркетинговых исследований.

Анализу опыта практического применения рассматриваемой модели в некоторых из этих сфер будет посвящена вторая статья данного цикла. Она в меньшей степени затрагивает теоретические вопросы, фокусируясь на представлении и анализе значительного объема уникальных эмпирических данных.

\section{2. Электоральные и маркетинговые исследования: схожие проблемы, единый источник.}

Для любой из социальных наук способность к предсказанию поведения человека является наивысшей точкой развития теории, а его точность - единственным

\footnotetext{
1 Это никак не ставит под сомнение ценность обширной дискуссии о современных проблемах эмпирической и теоретической социологии. В частности, вопросов онтологических изменений в мире, своего рода "деструктурации", социального дерегулирования и падения роли структур, объективно приводящих к снижению прогнозируемости поведения. В качестве только одного из характерных примеров можно назвать «К вопросу о приближающемся конце социологии" [Vandenberghe, Fuchs, 2019]. Однако это - самостоятельная вселенная, обсуждение которой выходит далеко за рамки настоящей статьи.
} 
надежным критерием действенности соответствующей модели. В этом смысле своевременное предвидение, например, тенденций политического и экономического развития, социальных беспорядков и экономических кризисов, является важнейшей общественной функцией рассматриваемых научных дисциплин. Несмотря на значительный прогресс, положение ни в одной из них в данной области нельзя признать удовлетворительным. Тем более актуальным представляется эффективный обмен наработанными достижениями, который, хотя и не имеет никаких методологических препятствий все еще остается исчезающе редким явлением.

В настоящей статье предпринимается попытка проанализировать возможность применения некоторых передовых наработок, полученных в последние годы на материале электоральной социологии в маркетинговых исследованиях. Эти частные дисциплины избраны исходя из того соображения, что именно в них накоплен наибольший опыт предсказания поведения людей. В одном случае - в качестве избирателей, в другом - в качестве потребителей. В обеих из них достаточно оперативно проверяются применяемые методы и наличествует довольно большое пространство для экспериментальной работы.

Немаловажно также, что как раз в силу открытости оперативной и достоверной проверке прогностической силы своих моделей именно две эти дисциплины подвергаются в последние годы довольно жесткой критике. Например, совсем недавно электоральная социология потерпела ряд болезненных поражений, выдав череду ошибочных прогнозов результатов выборов, часть из которых породила весьма чувствительные упреки в адрес отрасли в целом ${ }^{2}$. В качестве таковых кейсов обычно указывают на следующие:

- парламентские выборы 2015 года в Великобритании;

- выборы в кнессет 2015 года в Израиле;

- референдум 2015 года в Греции;

- президентские выборы 2015 года в Беларуси;

- референдум по Brexit 2016 года в Великобритании;

- президентские выборы 2016 года в США;

- парламентские выборы 2017 года в Великобритании.

Ошибки прогноза на выборах в парламент Великобритании в 2015 г. и во время избрания президента США в 2016 г. оказались настолько резонансными, что были организованы отраслевые расследования, призванные выяснить, как могло получиться, что ошиблись не отдельные исследовательские агентства, а вся отрасль в целом. Результаты данных расследований весьма показательны, итоговый вывод заключается в том, что все было сделано правильно и улучшить точность

\footnotetext{
2 Пресс-секретарь Барака Обамы Джош Эрнест: «Почти каждый опрос общественного мнения о предпочтениях американских избирателей перед выборами президента 8 ноября оказался ошибочным». См.: Белый дом признал ошибочность опросов о победе Клинтон //BBC: Русская служба новостей. 2016. 10 ноября. URL: http://www.bbc.com/ russian/news-37931882 (дата обращения: 12.08.2021). Влиятельный исследовательский центр сообщал на своем сайте: «Результаты президентских выборов во вторник оказались сюрпризом почти для каждого... Как опросы могли настолько сильно ошибаться?» См. Mercer A., Deane C., McGeeney K. Why 2016 Election Polls Missed their Mark? //Pew Research Center. 2016. URL: https://www.pewresearch.org/fact-tank/2016/11/09/why-2016-election-polls-missedtheir-mark (дата обращения: 13.08.2021). См. также: Whiteley P. Four Reasons Why the Polls Got the U.S. Election Result so Wrong// Newsweek. 2016. November 14. URL: http://www.newsweek.com/polls-2016-us-elections-trump-potushillary-clinton-520291 (дата обращения: 12.08.2021).
} 
прогноза практически невозможно. В частности, указывалось на следующие факторы, которые могут влиять на качество прогноза, но находятся вне контроля исследователя:

1. Ошибки в конструировании и реализации выборки: остаются неохваченными избиратели, проживающие за пределами избирательного округа, но голосующие удаленно. Или, наоборот, опрашиваются лица, проживающие на территории округа, но не являющиеся избирателями.

2. Проблемы достижимости: сторонники одного из кандидатов представляют аудиторию, традиционно имеющую более высокий/низкий коэффициент отказов от участия в исследовании.

3. Проблема «последующего сдвига»: на момент проведения опроса избиратели имеют одно мнение, а позже меняют его. В качестве частного случая данной проблемы может рассматриваться так называемый недостаток интроспекции: если респондент не слишком вовлечен в политический процесс, он может и не разобраться в том, к какому кандидату на самом деле склоняются его симпатии, и совершенно искренне ответить неправильно.

4. Проблема сознательного умолчания: респонденты сознательно лгут, отвечая на вопросы анкеты, а на избирательных участках голосуют в соответствии со своими действительными предпочтениями ${ }^{3}$.

По сути, и первое и второе расследования пришли к схожим выводам о том, что точность электорального прогнозирования фактически достигла своего предела и отрасли предвыборных опросов осталось только умыть руки. В несколько вольном пересказе резюме обоих отчетов может быть представлено следующим образом: “Мы не можем проверить, говорит ли нам респондент правду о своем выборе, и мы не можем гарантировать, что он не изменит свой выбор в промежуток времени между моментом опроса и моментом выбора. Раз так, мы не можем отвечать за расхождения между результатами исследования и результатами голосования". Данная позиция выглядит весьма уязвимой не только с политических позиций взаимодействия индустрии и общества, но и с чисто научных. Респондент не может быть виновным в том, что сообщает исследователю не то, что последний от него ожидает. Если исследователь безосновательно исходит из презумпции "компетентности и добросовестности" респондента, то это проблема исследователя, а не респондента.

Ожидаемо не все согласились с подобным подходом [Rogers, Aida, 2012], и многочисленные альтернативные подходы, стимулируемые неудовлетворенностью заказчиков электоральных исследований, получили дополнительный толчок развитию. В числе наиболее успешных из них обычно называют следующие:

- прогнозные рынки [Arrow et al., 2008; Atanasov et al., 2015];

- опросы об ожиданиях [Rothschild, Wolfers, 2011; Graefe, 2014];

- контент-анализ социальных сетей [Tumasjan et al., 2010];

- экономические модели [Tufte, 1978].

На этом фоне следует особо отметить надежды и связанные с ними беспрецедентные авансы, выдаваемые направлению «больших данных» [Burrows, Savage, 2014].

\footnotetext{
3 Результаты расследования по итогам парламентских выборов 2015 г. в Великобритании см. [Sturgis et al., 2016]. Результаты расследования по итогам президентских выборов 2016 г. в США см.: [Kennedy et al., 2016].
} 
Как бы там ни было, само разнообразие альтернативных подходов недвусмысленно указывает на неудовлетворенность потребителей рынка электоральных исследований и прогнозов имеющимся предложением, по крайней мере в части традиционных методов, основанных на опросах избирателей, хотя последние и остаются доминирующими как в академической, так и в прикладной сфере [Erikson, Panagopoulos, Wlezien, 2004; Newport, 2008; Perry, 1973, 1979; Vermunt, Magidson, 2005]. В целом же картина настолько неоднозначна, что уже в первой декаде наступившего века некоторые авторы находили возможным ставить вопрос о "кризисе эмпирической социологии" [Savage, Burrows, 2007].

Ситуация, которую мы наблюдаем в маркетинге, во многом сходна. В частности, с 1990-х годов указывалось на появление явных признаков недовольства заказчиков маркетинговых услуг их экономической эффективностью [Clancy, Krieg, 2000]. В последующие годы появилось значительное количество работ, раскрывающих эту тему более глубоко и предлагающих возможные альтернативы [Clancy, Krieg, McGarry Wolf, 2006], такие как нейромаркетинг [Lee, Broderick, Chamberlain, 2007; Fortunato, Giraldi, de Oliveira, 2014], экспериментальный маркетинг [Clancy, Krieg, McGarry Wolf, 2006], маркетинг, ориентированный на стоимость [Дойль, 2001] и т. п. В нашумевшей в свое время книге авторы пишут применительно к результатам опросов потребителей: «Не верьте, что люди будут поступать так, как они говорят" [Clancy, Krieg, 2000: 176]. Выход же ученые видят в более полном описании мотивов потребителей, в частности за счет комплексного учета всех трех классических компонент установки [ibid.: 122], в принципиальном отказе от выявления намерений в пользу изучения реального поведения потребителя в различных ситуациях, в том числе и специально моделируемых исследователем [Clancy et al., 2006].

Рассмотренные факты довольно определенно указывают на общий характер проблем, с которыми примерно в одинаковое время столкнулись как электоральная социология, так и маркетинг. Однако это сходство в том виде, в котором оно представлено выше, внешнее. Можно ли считать, что отмеченные дисфункции разных дисциплин, близкие по форме, имеют также и сходные основания? Имеются ли и достаточно близкие фундаментальные причины, открывающие, помимо прочего, возможности для корректного проецирования опыта одной дисциплины на предметное и методическое пространство другой?

Представляется, что ответ на эти вопросы должен быть положительным. Теоретической предпосылкой для возможности корректного обмена методологическими и методическими наработками является признание обеими дисциплинами поведения человека в качестве материального носителя предметов своего изучения. По данному вопросу обе дисциплины ${ }^{4}$ имеют сходные, иногда сближающиеся даже до степени тождественности представления.

Чтобы убедиться в этом, достаточно рассмотреть господствующую в социологических моделях, связанных с изучением поведения, теорию обдуманного действия / теорию запланированного поведения (ТОД/ТЗП) [Fishbein, Ajzen, 2011], которая основана на интуитивно-очевидном допущении, что действия человека опреде-

\footnotetext{
4 По крайней мере-в рамках направлений "классической социологии", "неоклассической экономики" и "неоинституциональной экономики".
} 
ляются ожиданиями их последствий. Чем более желательным представляется субъекту ожидаемый результат действия, тем выше мотивация его совершить и тем больше, при прочих равных условиях, вероятность того, что действие будет совершено (см. рис. 1).

Рис. 1. Классическая (однокомпонентная) модель прогнозирования поведения ТоД/ТЭП

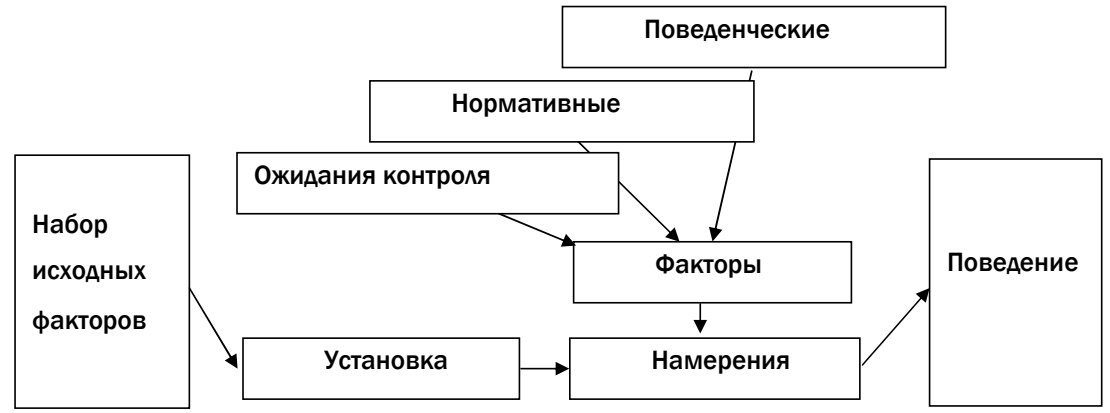

Такое понимание совокупности ожиданий в отношении будущего действия сами авторы ТОД/ТЗП называют моделью установки на ожидаемую полезность ("expectance-value model of attitude») [ibid.: 96]. Вполне очевидно, что социологическая модель установки на ожидаемую полезность полностью соответствует сложившемуся пониманию “экономического поведения", принятому в экономической науке [Walras, 1954; Friedman, Savage, 1948]. По сути, "обоснованное поведение" Фишбейна - Айзена является ничем иным, как частным случаем «экономического поведения". Если это так, то необходимой предпосылкой дальнейшего анализа будет обзор фундаментальных ограничений применимости моделей “экономического поведения".

В экономической науке фундаментальными критериями применимости модели "экономического поведения" являются следующие допущения:

- индивиды стремятся к "максимизации полезности";

- индивиды имеют рациональные предпочтения исходов событий;

- индивиды действуют в своих интересах, пытаясь опереться на максимально полную информацию [Weintraub, 2007].

Как известно, данные допущения не безусловны даже применительно к деятельности экономических субъектов и тем более неочевидны - для всех прочих сфер, где модель “экономического поведения" принимается в качестве подразумеваемой аксиомы.

В частности, Дж. Тинтнер показал, что “максимизация полезности" недостижима по фундаментальным основаниям, так как решения экономического/социального агента порождают не единственный исход, а вероятностные интервалы исходов, которые могут пересекаться [Tintner, 1941a, 1941b, 1942]. Р. Кокс [Cox, 2001], М. Дюпре и Ф. Типлер [Dupré, Tipler, 2009] поставили под сомнение теоретические основания модели максимизации полезности М. Фридмана - Л. Саважа, а М. Аллэ [Allais, 1953; Allais, Hagen, 1979] и Д. Эллсберг [Ellsberg, 1961] получили 
эмпирический материал, указывающий на обширные области поведения, где допущение о максимизации полезности не работает. Нобелевский лауреат Д. Канеман и А. Тверски показали, что даже в условиях удовлетворительного обеспечения исходными данными рациональные решения могут отвергаться по ряду оснований, имеющих психологическую природу [Tversky, Kahneman, 1992; Kahneman, 2011].

Закономерным образом открытие Д. Канемана и А. Тверски привело к появлению и бурному развитию альтернативных истолкований движущих сил поведения человека. В частности, в рамках "поведенческой экономики" активно разрабатываются модели "эвристического поведения" и "ограниченной рациональности", которые, как это достоверно установлено, имеют место в практической деятельности, но в полной мере пока не описаны [Goldstein, Gigerenzer, 2002; Kahneman, 2003; Shah, Oppenheimer, 2008; Талер, 2017].

Таким образом, в экономической науке наблюдается не только признание системных признаков эрозии конвенциональных теоретических парадигм, но и предпринимаются попытки их переосмысления и даже радикальной ревизии. С точки зрения предмета рассмотрения данной статьи этот процесс имеет весьма существенное значение, так как указывает на универсальный характер попыток выйти за пределы ограничений, задаваемых моделями «экономического поведения" ${ }^{5}$. Экономическая наука демонстрирует тот же вектор развития, который на протяжении последних десятилетий вызревает в рамках микросоциологии и социологических теорий действия. Более того, в социологии имеются также и некоторые, как представляется, ценные наработки в области прикладного использования идей преодоления прокрустова ложа «экономического поведения" в части понимания и прогнозирования социального действия.

Таким образом, между социологическим и экономическим подходом к изучению поведения человека имеется существенное сходство, локализуемое в области базовых допущений, то есть в самой основе всех дельнейших теоретических построений. В этом фундаментальном сходстве кроется, помимо прочего, и единая предпосылка характерных для обеих научных дисциплин проблем в области изучения и эффективного прогнозирования поведения человека, а именно - чрезмерное огрубление мотивов человека в модели "экономического поведения", развитие которой в направлении расширения принимаемых во внимание мотивов постепенно начинает рассматриваться как веление времени.

\section{3. От "экономического поведения" к "двухкомпонентной модели"}

Представленные выше аргументы в пользу наличия многочисленных ограничений использования модели "экономического поведения" приводят нас к выводу, что корректное применение этой модели и ее производных как к сфере электорального поведения, так и к сфере маркетинга должно опираться на признание ненулевой вероятности того, что поведение одних избирателей/покупателей соответствует критериям "экономического поведения", а поведение других не соответствует. Если это так, тогда использование модели "экономического поведения" в этих сферах всегда содержит риски неполноты описания факторов,

\footnotetext{
5 Невозможно не отметить также и бесспорные достижения в этой области, полученные в последнее время в социальной психологии и нейроэкономике.
} 
определяющих действия, в отношении которых строится прогноз. Здесь встает задача восполнить дефицит информации для случаев, когда выбор избирателей не полностью описывается моделью «экономического поведения". Как уже отмечалось ранее [Chernozub, 2020a], данную модель отличает сознательное абстрагирование от внерассудочных влияний. М. Фишбейн и А. Айзен пишут: «В целом общее настроение и эмоции рассматриваются в нашей модели прогнозирования поведения как фоновый фактор <...> даже если они влияют на некоторые представления, эти влияния могут быть не настолько сильными, чтобы менять установки, воспринятые нормы, нормативные ожидания со стороны окружающих" [Fishbein, Ajzen, 2010: 248]. Сами же представления служат рассудочной основой поведения в целом: «люди определяют себе цели, чтобы поступать разумно, обоснованно, непротиворечиво, иногда даже автоматически, исходя из того, что они сами думают о своих поступках" [ibid.: 24]. Авторы модели подчеркивают, что поведение в их модели определяется как обоснованное не потому, что оно "всегда логично и рационально", а потому что в представлениях, в соответствии с которыми субъект пытается достичь желаемого результата, имеется рассудочная основа (cognitive foundation) поведения [ibid.: 24].

Подобное абстрагирование от эмоций и других внерассудочных факторов вполне оправдано для модели “экономического поведения", однако если мы видим, что такая модель не может исчерпывающе описывать поведение человека, возникает потребность дополнить факторы, лежащие в пределах сознания человека, факторами, также оказывающими влияние на поведение человека, но лежащими вне такой сферы [Arcuri et al., 2008; Choma, Hafer, 2009; Greenwald et al., 2009a, 2009b].

В рамках сформировавшегося в последние годы консенсуса первые стало принято называть эксплицитными, а вторые - имплицитными факторами.

Эксплицитные факторы осознаются человеком и, при наличии его желания, могут быть достоверно сообщены окружающим, например, в ходе электорального или маркетингового опроса. Имплицитные, напротив, скрыты от внешнего наблюдения, так как либо не осознаются самим их носителем, либо скрываются им от внешнего мира, а иногда - и от самого себя [Fazio, Olson, 2014]. Как видно из представленного определения, структура имплицитных мотивов весьма неоднородна, однако в настоящее время они продолжают рассматриваться как единая категория. Поэтому в рамках настоящей статьи мы также не будем затрагивать проблему внутренней структуры имплицитных факторов, рассматривая их как сущностно единую противоположность эксплицитным.

Принятие гипотезы о наличии иных, лежащих вне рационального поиска оптимальных решений "максимизации полезности" имплицитных факторов, обуславливающих либо, по крайней мере, влияющих на осуществление реального действия, приводит нас в область структурной теории установки.

Структурная теория установки постулирует наличие нескольких относительно независимых компонентов, к классификации которых имеется несколько подходов. Традиционная модель различает "когнитивную", “аффективную" и "конативную" компоненты [Katz, 1960; Smith, 2003], в то время как некоторые современные авторы предпочитают говорить об “эксплицитных" и "имплицитных" компонентах, которые формируют целостное отношение, определяют намерения, а в конечном 
счете - и, собственно, реальные действия человека. М. Перуджини классифицировал современные структурные модели установки, сведя их к трем основным подходам [Perugini, 2005]:

1. "Полная независимость". В рамках этого подхода эксплицитные и имплицитные компоненты рассматриваются как не взаимодействующие друг с другом и более того - как влияющие на различные типы поведения. Считается, что эксплицитные факторы влияют на преднамеренное поведение, контролируемое сознанием, в то время как имплицитные - на импульсивное поведение, которое не контролируется сознанием человека. Соответственно, чтобы правильно спрогнозировать поведение первого типа, необходимо опираться на эксплицитные, а второго типа - на имплицитные факторы.

2. “Дополнительность". Оба компонента рассматриваются не как самостоятельные явления, имеющие свою природу, закономерности, траектории развития и т. д., но как проявления одного-единственного отношения, не вполне совпадающие друг с другом только из-за различий в методах измерения. В этом смысле результаты различных методов измерения дополняют и уточняют друг друга, позволяя представить установку в наиболее комплексной, полной форме.

3. “Взаимодействие”. Этот подход объединяет тех, кто, подобно сторонникам "независимости", считает эксплицитную и имплицитную компоненты установки самодостаточными явлениями, но не считает, что они определяют каждый свой "собственный" вид поведения. Фактическое действие человека в рамках данного подхода рассматривается как результат взаимодействия компонент.

В контексте данной классификации рассматриваемая в настоящей статье модель прогнозирования базируется на третьем подходе - "взаимодействии", рассматривающем эксплицитные и имплицитные компоненты установки как самостоятельные явления, имеющие свою природу и влияющие на поведение человека через некую "результирующую" их взаимодействия. Особая механика этого взаимодействия формируется согласованным или несогласованным статусом установки.

В свою очередь, в пределах структурной теории установки потенциальная несогласованность последней рассматривается в рамках нескольких общих подходов.

1. Несогласованность отношения и поведения [Heider, 1958; Himmelfarb, Eagly, 1974; Kiesler, Collins, Miller, 1969; O’Keefe, 1990].

2. Несогласованность внутренних структурных элементов когнитивной компоненты [Festinger, 1957; Festinger, Carlsmith, 1959].

3. Противоречивость различных (например, сознательных и бессознательных) компонент самой установки [Rosenberg, 1956; Rosenberg et al., 1960].

Последний из перечисленных подходов к анализу состояний несогласованности установки на данный момент наименее изучен. Эксперименты М. Розенберга, выявив явление рассогласованности компонентов установки как таковое, не дали никакой информации о влиянии этой рассогласованности на поведение. Частично данная проблематика была затронута М. Роккато и К. Зогмайстер [Roccato, Zogmaister, 2010]. В своем эксперименте на основе IAT ${ }^{6}$ они попытались

\footnotetext{
6 Implicit Attitude Test. См.: https://implicit.harvard.edu/implicit/takeatest.html; https://www. projectimplicit.net/ (дата обращения: 12.08.2021).
} 
сопоставить политические установки и поведение респондентов при голосовании. Однако данные, описывающие непосредственно само электоральное поведение, были собраны с помощью самоотчетов респондентов. Это означает, что данная попытка, скорее всего, не может рассматриваться как полноценная проверка связи между установкой и поведением. Подобное предположение тем более имеет право на существование, если учесть данные о несоответствии самоотчетов действиям, полученные как раз на материале электорального поведения Т. Роджерсом и М. Аидой [Rogers, Aida, 2012].

Тем не менее М. Роккато и К. Зогмайстер пришли к ценному для нашего анализа выводу: «В частности, из представленных данных выяснилось, что взаимодействие между имплицитными и эксплицитными компонентами установки может задерживать (когда они не согласованы) или облегчать (когда они согласованы) принятие решения; важно, однако, что представленные данные предполагают, что окончательное поведение (например, участие в голосовании как таковое и содержание поданного бюллетеня) не зависит от взаимодействия между имплицитными и эксплицитными политическими предпочтениями" [Roccato, Zogmaister, 2010: 272].

Вторая часть предлагаемой ими интерпретации кажется несколько противоречивой. Если статус согласованности/несогласованности установки влияет на решение действовать, то трудно представить, как это влияние не может повлиять на само "конечное" действие. Например, логично предположить, что избиратель, имеющий согласованную установку в отношении "своего" кандидата, с большей вероятностью явится на избирательный участок и проголосует, чем (при прочих равных условиях) тот избиратель, чьи компоненты установки конфликтуют друг с другом. Результаты исследований М. Перуджини также вполне поддерживают это предположение: «Эмпирические данные подтверждают, что прогноз того, является ли человек курильщиком или нет, оказывается более точным в случаях, когда имплицитные и эксплицитные компоненты установки развернуты в одном и том же направлении..." [Perugini, 2005: 43]. «Ключевой посыл заключается в том, что имплицитные и эксплицитные компоненты установки могут взаимодействовать, влияя на поведение» [ibid.: 53].

В теоретическом аспекте это означает существенную перестройку исходной модели ТОД/ТЗП [Fishbein, Ajzen, 2011] за счет обогащения ее самостоятельным потоком воздействий на поведение за счет имплицитных факторов (выделены курсивом).

\section{Рис. 2. Двухкомпонентная модель прогнозирования поведения}

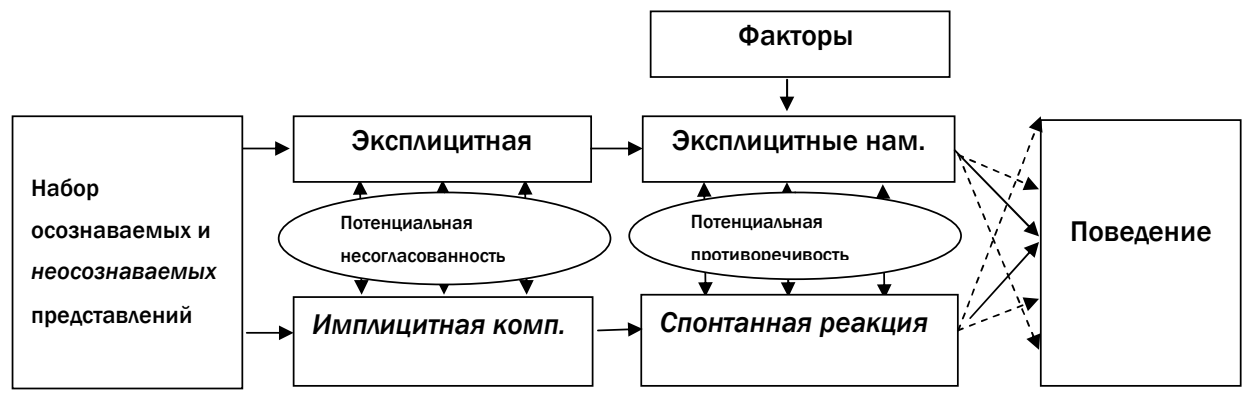


Убеждения - это совокупность базовых факторов, влияющих на формирование установки, которую, несмотря на то, что ее структура может быть более сложной, авторы модели обозначили как "убеждения" [ibid.: 96].

Факторы тоД/ТЗП - это воспринимаемые нормы, регулирующие поведение субъекта действия, ожидаемые им последствия своих действий и так называемые ожидания контроля, то есть его субъективная оценка степени добровольности совершения действия. Чем благоприятнее последствия действия, чем позитивнее поддержка этого действия со стороны формальных и неформальных норм референтных групп, чем глубже убежденность в добровольном характере принятого решения - тем выше вероятность совершения действия.

Эксплицитная компонента установки - осознанное отношение к объекту действия, которое может быть корректно сформулировано и сообщено окружающим.

Эксплицитные намерения - осознанные намерения, доступные для понятийного описания и критического анализа.

Имплицитная компонента установка - неосознаваемое отношение. Индивид может не замечать даже ее присутствия и тем более не понимать ни ее природы, ни ее силы или валентности. По данным Т. Уилсона и коллег [Wilson, Lindsey, Schooler, 2000], имплицитное отношение активизируется автоматически и может быть или не быть подавлено сознательными (эксплицитными) факторами.

Спонтанная реакция - это бессознательные побуждения к действию, “автоматически" порождаемые имплицитной установкой. Их иногда называют имплицитными намерениями, несмотря на то, что они, будучи бессознательными, безусловно, не являются "намерениями" в прямом смысле этого слова.

Поведение - это реальный акт, представляющий собой конечный результат взаимодействия имплицитных и эксплицитных факторов.

Представленная выше схема выглядит почти так же, как рефлексивная и импульсивная модель (RIM) [Strack, Deustch, 2004], однако, фокусируясь на социальных драйверах поведения, она рассматривает не только психические механизмы формирования действия, но и социальные факторы этого процесса.

Таковы теоретические основы рассмотрения более широкого по сравнению с классической моделью “экономического поведения" спектра факторов, определяющих действия человека. Практическая форма построения "Двухкомпонентной модели прогнозирования поведения", примеры и результаты использования ее в электоральных исследованиях представлены ниже. Однако прежде, чем обратиться к вопросам практического применения представленных выше теоретических построений, необходимо сделать важное отступление в область того, какими средствами измерялась собственно имплицитная компонента.

Как оказалось, задача эта весьма нетривиальна, поэтому в рамках многочисленных методических экспериментов ВЦИОМ автором был разработан, валидизирован и отьюстирован измерительный инструмент, получивший в конце концов наименование «Графический ассоциативный тест отношения" (ГАТО) [Chernozub, 2020a, 2020b]. 


\section{4. Двухкомпонентная модель: первые успехи в электоральном прогнозировании и некоторые уроки для маркетинга}

Двухкомпонентная модель поведения изначально предназначалась для прогнозирования результатов выборов. Начиная с 2015 г. было проведено большое количество исследований, где она стабильно демонстрировала лучшие по сравнению с традиционными моделями результаты. Для оценки преимуществ двухкомпонентной модели прогнозирования поведения (экспериментальной) мы поставили задачу сравнить получаемые с ее помощью результаты с результатами традиционных моделей прогнозирования, которые, таким образом, выступили в качестве контрольных.

Для решения этой задачи среди традиционных моделей нами было отобрано три наиболее распространенных, каждая из которых является общепризнанными инструментом прогнозирования результатов голосования. Это немаловажно в контексте предметного пространства данной статьи - они же в несколько измененной форме используются и для прогнозирования потребительского поведения.

Каждая из контрольных моделей основывается на том допущении, что выбор агентом варианта действия является результатом действия эксплицитных факторов, приводящих к формированию осознанного намерения. Экспериментальные модели принимали во внимание как рациональные (эксплицитные), так и внерациональные (имплицитные) факторы поведения.

Модель "намерение". В "контрольном" режиме модель "намерение" означает принятие положительного прогноза голосования за соответствующего кандидата для респондентов, выбравших этого кандидата, при ответе на вопрос: «Если бы выборы состоялись в следующее воскресенье, за кого из этих кандидатов Вы бы проголосовали?» ${ }^{7}$ В маркетинге аналогом данного вопроса служит вопрос: «Какую из приведенных ниже марок вы намереваетесь купить во время ближайшего похода в магазин?", на практике применяющийся, конечно, в большом количестве разнообразных модификаций, отражающих специфику задач каждого конкретного исследования.

Модель "намерение + голосование" (likelihood to vote / vote intention - LVVI) дает такой прогноз для тех, кто одновременно заявил о своем намерении голосовать за конкретного кандидата и подтвердил свое намерение пойти на избирательный участок ${ }^{8}$. В маркетинговых исследованиях подобная конструкция для прогноза объема сбыта на определенный период используется в форме, когда вопрос о намерении купить конкретную марку некоего товара сопровождается вопросом о времени планируемого визита в магазин.

Модель "подтвержденное намерение" обеспечивает позитивный прогноз для тех, кто заявил о своем намерении голосовать за конкретного кандидата и в то же время положительно ответил на вопрос: "Является ли Ваше решение окончательным или оно все еще может измениться?"

Модель LVVI подтвержденная. Наконец, последняя модель использует все три фильтра одновременно. Иными словами, положительный прогноз в отноше-

\footnotetext{
7 Предлагается список баллотирующихся кандидатов, имеются варианты “ни за кого из этого списка", «затрудняюсь ответить", "не хотел бы отвечать на этот вопрос".

8 Вопрос модели «намерение" дополняется вопросом: «Намереваетесь ли Вы принять проголосовать на выборах ?". Имеются варианты "еще не решил", "затрудняюсь ответить", “не хотел бы отвечать на этот вопрос".
} 
нии голосования за определенного кандидата сохраняется только для тех, кто подтвердил свою готовность принять участие в голосовании, указал кандидата, за которого намеревается голосовать, и подтвердил, что это его окончательное решение.

В “экспериментальном" режиме каждая из рассмотренных моделей дополнялась имплицитным фильтром отношения: положительный прогноз голосования сохранялся только для тех, кто продемонстрировал положительное имплицитное отношение к соответствующему кандидату по данным теста ГАТО. Таким образом, на основании каждой из этих моделей был построен прогноз в "контрольном" (на основе только эксплицитной компоненты) и "экспериментальном" (с учетом двух компонент - и эксплицитной, и имплицитной) режимах.

В таблице 1 представлены данные усредненных отклонений прогнозов различных моделей от фактических результатов, позволяющие дать сравнительную оценку эффективности применения традиционных и экспериментальных моделей. Для оценки относительной точности результатов, полученных при помощи каждой из них, мы вычли значения фактических результатов соответствующих кандидатов из полученных для них прогнозных значений. Затем модуль полученного таким образом отклонения нормировался, делясь на фактический результат для данного кандидата. В итоге в таблице 1 представлены нормированные отклонения, усредненные по всем кандидатам, получившим 5 \% и более голосов на соответствующих выборах (четыре крупнейших партии на выборах в Государственную Думу и три кандидата - на президентских выборах). Таким образом, данные по семи кандидатам позволили построить 28 контрольных и 28 экспериментальных моделей, формируя массив из 56 альтернативных прогнозных значений, доступных для проведения сравнительного анализа.

Таблица 1. Сравнительный анализ усредненных отклонений экспериментальных и контрольных моделей (п. п., данные предвыборных опросов ВЦИОМ 2016-2018 гг.)

\begin{tabular}{|c|c|c|c|c|}
\hline Прогнозные модели & Намерение & LVVI & $\begin{array}{l}\text { Намерение } \\
\text { подтв. }\end{array}$ & LVVI подтв. \\
\hline \multicolumn{5}{|l|}{ Выборы в Государственную думу, 2016 г. } \\
\hline $\begin{array}{l}\text { Контрольный режим (только эксплицит- } \\
\text { ные факторы) }\end{array}$ & 25,3 & 24,9 & 28,9 & 16,6 \\
\hline $\begin{array}{l}\text { Экспериментальный режим (эксплицит- } \\
\text { ные + имплицитные факторы) }\end{array}$ & 20,7 & 18,0 & 11,8 & 9,4 \\
\hline $\begin{array}{l}\text { Улучшение прогноза в эксперименталь- } \\
\text { ных моделях }\end{array}$ & 4,6 & 6,9 & 17,1 & 7,2 \\
\hline \multicolumn{5}{|l|}{ Выборы Президента РФ, 2018 г. } \\
\hline $\begin{array}{l}\text { Контрольный режим (только эксплицит- } \\
\text { ные факторы) }\end{array}$ & 23,4 & 9,6 & 25,3 & 53,2 \\
\hline $\begin{array}{l}\text { Экспериментальный режим (эксплицит- } \\
\text { ные + имплицитные факторы) }\end{array}$ & 21,3 & 8,5 & 26,1 & 26,6 \\
\hline $\begin{array}{l}\text { Улучшение прогноза в эксперименталь- } \\
\text { ных моделях }\end{array}$ & 2,1 & 1,1 & $-0,8$ & 26,6 \\
\hline
\end{tabular}


Как следует из данных таблицы 1, среднее нормированное отклонение прогноза контрольных моделей от фактического результата на выборах в Государственную думу 2016 г. составило от 16,6 п. п. для модели "LVVI подтвержденная", до 28,9 п. п. для модели «Намерение подтвержденное». Для экспериментальных моделей данный показатель оказался в диапазоне от 9,4 п. п. для модели "LVVI подтвержденная" до 20,7 п. п. для модели "Намерение». Улучшение прогноза за счет перехода от однокомпонентной к двухкомпонентной модели факторов поведения составило от 6,9 п. п. до 17,1 п. п. для моделей «LVVI» и «Намерение подтвержденное", соответственно.

Для прогнозов результатов президентских выборов 2018 г. ошибка контрольных моделей составила от 9,6 п. п. для “LVVI" до 53,2 п. п. для “LVVI подтвержденная". Ошибка экспериментальных моделей— от 8,5 п. п. для "LVVI" до 26,6 п. п. для "LVVI подтвержденная". Улучшение прогноза составило от 1,1 п. п. до 26,6 п. п. В одном случае зафиксировано ухудшение прогноза на 0,8 п. п., которые на общем фоне всей совокупности полученных результатов выглядят относительно незначительной величиной.

Как видно из представленных данных, эмпирические исследования 20162018 гг. выявили достаточно устойчивую закономерность повышения точности прогнозов для всех моделей при переходе от традиционного к экспериментальному режиму их использования. Поскольку единственным параметром, отличавшим экспериментальные модели от контрольных, являлось использование данных о состоянии имплицитной компоненты установки, данное улучшение невозможно приписать никакому иному фактору. Более того, позднейший анализ показал, что точность прогноза, выполненного только на основе имплицитной компоненты, оказывается заметно ниже точности прогноза, выполненного на основе только эксплицитной компоненты, и что собственно улучшение точности прогноза дает именно сочетание обеих компонент в одной прогнозной модели. Все эти факты явным образом указывают на синергетический характер возникающего эффекта, что, в свою очередь, позволяет говорить о наличии у системы экспериментальных моделей свойства эмерджентности.

Раз двухкомпонентная модель факторов поведения человека работает применительно к электоральному поведению, будет ли она работать также и в отношении потребительского поведения? Насколько мы могли убедиться, никаких теоретических препятствий для этого не просматривается. В практическом плане также имеются первые, пусть пока фрагментарные, но вполне обнадеживающие результаты. В частности, совместно с одним из ведущих маркетинговых агентств нашей страны мы провели пилотный проект на рынке FMCG. Обобщение результатов этого прикладного исследования - тема самостоятельной статьи. Тем не менее уже сейчас вполне уверенно просматриваются некоторые очевидные области применения двухкомпонентной модели поведения в прикладном маркетинге. Дадим им краткую характеристику.

1. Прогноз продаж. Точно так же, как и на политическом, на товарном рынке покупатели каждого конкретного бренда образуют "ядро" и концентрические круги "периферии" его покупательской аудитории. Таких кругов может быть несколько, они движимы разными мотивами и поэтому закономерно различаются 
вероятностью покупки целевого бренда. Правильно оценивая объем этих групп и вероятность их переключения в каждом цикле покупок, можно (и нужно) делать более точные прогнозы объемов продаж.

2. Маркетинговая стратегия. Правильная оценка состояния указанных выше генеральных факторов продаж (объем и вероятность покупки различных групп покупательской аудитории) открывает возможности для сознательного управления ими. В частности, на основе более точного сегментирования рынка, адекватной оценки инсайтов собственного бренда и брендов-конкурентов, учета и грамотного использования культурных особенностей потребителей.

3. Сегментирование рынка. В норме рядовой потребитель не в состоянии достоверно отрефлексировать реальные мотивы своего потребительского поведения. Поэтому его ответы на "когнитивные" (пропускаемые через сознание) вопросы анкеты маркетингового исследования заведомо искажены эффектами "недостатка интроспекции", а часто и "сознательного искажения". Дополнение измерения эксплицитных мотивов потребителя выявлением имплицитных мотивов позволяет сформировать объемную и более реалистичную картину сил, движущих его покупательским поведением, и провести корректное сегментирование рынка.

4. Бренд-инсайт. Часто параметры и даже измерения («пространства различения"), в рамках которых потребители различают бренды, достоверно неизвестны владельцам последних. Причина та же: потребители сами толком не знают, почему они на самом деле предпочитают один бренд другому. Что в действительности бренд "говорит" потребителю? А что "говорят" бренды конкурентов? Использование имплицитной компоненты позволяет внести ясность в этом вопросе. В особенности в тех случаях, когда дело касается товарных категорий и брендов, апеллирующих прежде всего к эмоциональной сфере восприятия потребителей.

5. Культурные факторы спроса. Часто культурные нормы и ценности настолько глубоко "Въедаются" в сознание и подсознание человека, что он сам не осознает силу их влияния на свое поведение. В политической социологии мы используем инструмент "карта бессознательного", который в наглядном виде показывает, как интересующие нас объекты реального мира "ложатся" на систему глубинных предпочтений человека. Это открывает возможности сделать продуктовое и ценовое предложение более точным, а послание бренда - более понятным, спотыкающимся о меньшее количество барьеров, существование которых часто не осознается и самим потребителем.

\section{Заключение}

Проведенный анализ показал, что в электоральной социологии появились некоторые наработки, заимствование которых маркетингом выглядит довольно перспективным.

Во-первых, данные наработки действительно демонстрируют эффективность, причем делают это весьма устойчиво. В настоящее время накоплено огромное количество фактов, подтверждающих закономерный характер их относительно более высоких результатов. Иными словами, изучение вопроса об использовании передовых методов политической социологии в маркетинге целесообразно. 
Во-вторых, для такого заимствования не обнаруживается фундаментальных теоретических препятствий. Методологической основой обеих дисциплин выступает модель "экономического поведения" как основы деятельности человека. "Человек экономический", в рамках своей деятельности "максимизирующий полезность",- это аксиоматическое допущение лежит в основе господствующих научных парадигм как в социологии, так и в экономике.

В-третьих, уже сейчас обнаруживается целый перечень предметных областей маркетинга, где использование методического багажа электоральных опросов и электорального прогнозирования оказывается очевидно уместным. К таким областям относятся прогнозирование спроса и продаж, сегментирование потребителей, разработка новых брендов, конструирование инсайтов, бренд-лифтинг, ценовая политика, продуктовая стратегия и стратегия продвижения, маркетинговая стратегия в целом.

Нет сомнений в том, что массовое внедрение рассмотренных моделей и методов в практику маркетинга выявит какие-то затруднения, которые мы пока упускаем из виду. Однако эти затруднения наверняка будут носить частный, технический, иными словами - вполне преодолимый характер. В этом, помимо прочего, нас убеждает опыт применения ГАТО в рамках уже упоминавшегося исследования конкурентной ситуации на одном из рынков FMCG. Как оно проходило и к каким выводам привело совместную группу исследователей - в следующей статье.

\section{Список литературы (References)}

Дойль П. Маркетинг, ориентированный на стоимость. СПб. : Питер, 2001.

Doyle P. (2001) Marceting Strategies for Corporate Growth and Shareholder Value. St. Petersburg: Piter. (In Russ.)

Талер Р. Новая поведенческая экономика. Почему люди нарушают правила традиционной экономики и как на этом заработать. М. : ЭКСМО, 2017.

Thaler R. (2017) Misbehaving: The Making of Behavioral Economics. Moscow: EKSMO. (In Russ.)

Allais M. (1953) Le Comportement de l'Homme Rationnel Devant Le Risque: Critique des Postulats et Axiomes de L'Ecole Americaine. Econometrica. Vol. 21. No. 4. P. 503546. https://doi.org/10.2307/1907921.

Allais M., Hagen O. (eds.) (1979) Expected Utility Hypothesis and the Allais Paradox. Dordrecht: Reidel.

Arcuri L., Castelli L., Galdi S., Zogmaister C., Amadori A. (2008) Predicting the Vote: Implicit Attitudes as Predictors of the Future Behavior of Decided and Undecided Voters. Political Psychology. Vol.29. P. 369-387.

Arrow K., Forsythe R., Gorham M., Hahn R. (2008) The Promise of Prediction Markets. Science. Vol. 320. P. 877-878. https://doi.org/10.1126/science.1157679.

Atanasov P., Rescober Ph., Stone E., Swift S., Servan-Schreiber E., Tetlock Ph.E., Ungar L., Mellers B. (2015) Distilling the Wisdom of Crowds: Prediction Markets 
Versus Prediction Polls. Academy of Management Proceedings. No. 1. https://doi. org/10.5465/AMBPP.2015.15192abstract.

Burrows R., Savage M. (2014) After the Crisis? Big Data and the Methodological Challenges of Empirical Sociology. Big Data \& Society. https://doi.org/10.1177/ 2053951714540280.

Chernozub O. L. (2020a) Implicit Factors and Voting Behaviour Inconsistency: From Theoretical Concept to Empirical Phenomenon. Monitoring of Public Opinion: Economic and Social Changes. No. 4. P. 17-40. https://doi.org/10.14515/ monitoring.2020.4.1584.

Chernozub O. L. (2020b) Implicit Factors and Voting Behavior Inconsistency: From an Attitude to Behavior Monitoring of Public Opinion: Economic and Social Changes. No. 5. P. 71-89. https://doi.org/10.14515/monitoring.2020.5.1796.

Choma B. L., Hafer C. L. (2009) Understanding the Relation Between Explicitly and Implicitly Measured Political Orientation: The Moderating Role of Political Sophistication. Personality and Individual Differences. Vol. 47. P. 964-967.

Clancy K., Krieg P. (2000) Counterintuitive Marketing: Achieving Great Results Using Uncommon Sense. New York, NY: Free Press.

Clancy K., Krieg P., McGarry Wolf M. (2006) Market New Products Successfully: Using Simulated Test Market Technology. Lanham: Lexington Books.

Cox R. T. (2001) Algebra of Probable Inference. Baltimore: The Johns Hopkins University Press.

Dupré M. J., Tipler F. T. (2009) New Axioms for Bayesian Probability. Bayesian Analysis. Vol. 4. No. 3. P. 599-606. https://doi.org/10.1214/09-BA422.

Ellsberg D. (1961) Risk, Ambiguity, and the Savage Axioms. The Quarterly Journal of Economics. Vol. 75. No. 4. P. 643-669. https://doi.org/10.2307/1884324.

Erikson R., Panagopoulos C., Wlezien C. (2004) Likely (and Unlikely) Voters and the Assessment of Compaign Dynamics. Public Opinion Quarterly. Vol. 68. No. 4. P. 588601. https://doi.org/10.1093/poq/nfh041.

Festinger L. (1957) A Theory of Cognitive Dissonance. Evanston, IL: Row \& Peterson.

Festinger L., Carlsmith J. M. (1959) Cognitive Consequences of Forced Compliance. The Journal of Abnormal and Social Psychology. Vol. 58. No. 2. P. 203-210. https:// doi.org/10.1037/h0041593.

Fazio R., Olson M. (2014) The MODE model: Attitude-Behavior Processes as a Function of Motivation and Opportunity. In: Dual Process Theories of the Social Mind. New York: Guilford Press.

Fishbein M., Ajzen I. (2011) Predicting and Changing Behavior: The Reasoned Action Approach. New York; Hove: Psychology Press. 
Fortunato V. C. R., Giraldi J. M. E., de Oliveira J. H. C. (2014) A Review of Studies on Neuromarketing: Practical Results, Techniques, Contributions and Limitations. Journal of Management Research. Vol. 6. No. 2. P. 201-220.

Friedman M., Savage L. J. (1948) Utility Analysis of Choices Involving Risk. Journal of Political Economy. Vol. 56. No. 4. P. 279-304.

Goldstein D. G., Gigerenzer G. (2002) Models of Ecological Rationality: The Recognition Heuristic. Psychological Review. Vol. 109. No. 1. P. 75-90. http://dx.doi.org/ 10.1037/0033-295X.109.1.75.

Graefe A. (2014) Accuracy of Vote Expectation Surveys in Forecasting Elections. Public Opinion Quarterly. Vol. 78. No. S1. P. 204-232. https://doi.org/10.1093/poq/nfu008.

Greenwald A. G., PoehIman T. A., UhImann E. L., Banaji M. R. (2009a) Understanding and Using the Implicit Association Test: III. Meta-Analysis of Predictive Validity. Journal of Personality and Social Psychology. Vol. 97. No. 1. P. 17-41.

Greenwald A. G., Smith C. T., Sriram N., Bar-Anan Y., Nosek B. A. (2009b) Implicit Race Attitudes Predicted Vote in the 2008 U.S. Presidential Election. Analyses of Social Issues and Public Policy. Vol. 9. P. 241-253.

Heider F. (1958) The Psychology of Interpersonal Relations. Hoboken, NJ: John Wiley \& Sons. http://dx.doi.org/10.1037/10628-000.

Himmelfarb S., Eagly A. H. (1974) Orientations to the Study of Attitudes and Their Change. In: Himmelfarb S., Eagly A. H. (eds.) Readings in Attitude Change. New York, NY: John Wiley \& Sons. P. 2-49.

Kahneman D. (2003) Maps of Bounded Rationality: Psychology for Behavioral Economics. The American Economic Review. Vol. 93. No. 5. P. 1449-1475.

Kahneman D. (2011) Thinking, Fast and Slow. New York, NY: Farrar, Straus and Giroux. Katz D. (1960) The Functional Approach to the Study of Attitudes. Public Opinion Quarterly. Vol. 24. No. 2. P. 163-204. https://doi.org/10.1086/266945.

Kennedy C., Blumenthal M., Clement S., Clinton J., Durand C., Franklin Ch., McGeeney K., Miringoff L., Olson K., Rivers D., Saad L., Witt E., Wlezien Ch. (2016) An Evaluation of 2016 Election Polls in the U.S. AAPOR. URL: https://www.aapor.org/Education-Resources/ Reports/An-Evaluation-of-2016-Election-Polls-in-the-U-S.aspx (accessed: 14.08.2021).

Kiesler Ch.A., Collins B.E., Miller N. (1969) Attitude Change. A Critical Analysis of Theoretical Approaches. New York, N.Y.: John Wiley and Sons.

Lee N., Broderick A. J., Chamberlain L. (2007) What Is ‘Neuromarketing’? A Discussion and Agenda for Future Research. International Journal of Psychophysiology. Vol. 63. No. 2. P. $199-204$.

Newport F. (2008) Who are Likely Voters and When Do they Matter. Gallup. URL: http:// www.gallup.com/poll/109135/who-likely-voters-when-they-matte.aspx (accessed: 14.08.2021). 
O’Keefe D.J. (1990) Persuasion: Theory and Research. Newbury Park, CA: SAGE.

Perugini M. (2005) Predictive Models of Implicit and Explicit Attitudes. British Journal of Social Psychology. Vol. 44. No. 1. P. 29-45. https://doi.org/10.1348/ $014466604 \times 23491$.

Perry P. (1973) A Comparison of the Voting Preferences of Likely voters and Likely Nonvoters. The Public Opinion Quarterly. Vol. 37. No. 1. P. 99-109. https://doi.org/ 10.1086/268063.

Perry P. (1979) Certain Problems in Election Survey Methodology. Public Opinion Quarterly. Vol. 43. No. 3. P. 312-325. https://doi.org/10.1086/268524.

Roccato M., Zogmaister C. (2010) Predicting the Vote Through Implicit and Explicit Attitudes: A Field Research. Political Psychology. Vol. 31. No. 2. P. 249-274. https:// doi.org/10.1111/j.1467-9221.2009.00751.x.

Rogers T., Aida M. (2012) Why Bother Asking? The Limited Value of Self-Reported Vote Intention. Harvard Kennedy School of Government. Faculty Research Working Paper Series. https://dx.doi.org/10.2139/ssrn.1971846.

Rosenberg M.J. (1956) Cognitive Structure and Attitudinal Affect. The Journal of Abnormal and Social Psychology. Vol. 53. No. 3. P. 367-372. http://dx.doi.org/ 10.1037/h0044579.

Rosenberg M. J., Hovland C. I., McGuire W.J., Abelson R. P., Brehm J. W. (1960) Attitude Organization and Change: An Analysis of Consistency Among Attitude Components. Oxford: Yale University Press.

Rothschild D., Wolfers J. (2011) Forecasting Elections: Voter Intentions Versus Expectations. SSRN. URL: https://ssrn.com/abstract=1884644 (accessed: 14.08.2021).

Savage M., Burrows R. (2007) The Coming Crisis of Empirical Sociology. Sociology. Vol. 41. No. 5. P. 885-899. https://doi.org/10.1177/0038038507080443.

Shah A. K., Oppenheimer D. M. (2008) Heuristics Made Easy: An Effort-Reduction Framework. Psychological Bulletin. Vol. 134. No. 2. P. 207-222. https://doi. org/10.1037/0033-2909.134.2.207.

Smith M. B. (2003) For a Significant Social Psychology: The Collected Writings of M. Brewster Smith. New York, NY: New York University Press.

Strack F., Deutsch R. (2004) Reflective and Impulsive Determinants of Social Behavior. Personality and Social Psychology Review. Vol. 8. P. 220-247.

Sturgis P., Baker N., Callegaro M., Fisher S., Green J., Will J., Kuha J., Lauderdale B., Smith P. (2016) Report of the Inquiry into the 2015 British General Election Opinion Polls. London: Market Research Society and British Polling Council.

Tintner G. (1941a) The Pure Theory of Production under Technological Risk and Uncertainty. Econometrica. Vol. 9. No. 3/4. P. 305-311. https://doi.org/10.2307/ 1907199. 
Tintner G. (1941b) The Theory of Choice under Subjective Risk and Uncertainty. Econometrica. Vol. 9. No. 3/4. P. 298-304. https://doi.org/10.2307/1907198.

Tintner G. (1942) A Contribution to the Nonstatic Theory of Production. In: Studies in Mathematical Economics and Econontetrics. Chicago: University of Chicago Press. P. 92-109.

Tversky A., Kahneman D. (1992) Advances in Prospect Theory: Cumulative Representation of Uncertainty. Journal of Risk and Uncertainty. Vol. 5. No. 4. P. 297-323. https:// doi.org/10.1007/BF00122574.

Tufte E. (1978) Political Control of the Economy, Princeton, NJ: Princeton University Press.

Tumasjan A., Sprenger T. O., Sandner P. G., Welpe I. M. (2010) Predicting Elections With Twitter: What 140 Characters Reveal About Political Sentiment. Proceedings of the 4th International AAAI Conference on Weblogs and Social Media. AAAI Press. P. 178-185.

Vandenberghe F., Fuchs S. (2019) On the Coming End of Sociology. Canadian Review of Sociology / Revue canadienne de sociologie. Vol. 56. No. 1. P. 138-143. https:// doi.org/10.1111/cars.12238.

Vermunt J.K., Magidson J. (2005) Factor Analysis with Categorical Indicators: A Comparison between Traditional and Latent Class Approaches. In: van der Ark L. A., Croon M. A., Sijtsma K. (eds.) Quantitative Methodology Series. New Developments in Categorial Data Analysis for the Social and Behavioral Sciences. Mahwah, NJ: Lawrence Erlbaum Associates Publishers. P. 41-62.

Wilson T., Lindsey S., Schooler T. (2000) A Model of Dual Attitudes. Psychological Review. Vol. 107. No. 1. P. 101-126. https://doi.org/10.1037/0033-295X.107.1.101.

Walras L. (1954) Elements of Pure Economics, or The Theory of Social Wealth. Transl. by W. Jaffé. London: Allen and Unwin.

Weintraub E.R. (2007) Neoclassical Economics. The Concise Encyclopedia of Economics. URL: http://www.econlib.org/library/Enc1/NeoclassicalEconomics.html (accessed: 14.08.2021). 